\title{
Telemetry Data Transmission Method Based on Wireless Multi-Hop Network
}

\author{
Zhida $\mathrm{Li}^{1, \mathrm{a}}$, Ping Song ${ }^{1, \mathrm{~b}}$ and Chuangbo $\mathrm{Hao}^{1, \mathrm{c}}$ \\ ${ }^{1}$ Key Laboratory of Biomimetic Robots and Systems, Beijing Institute of Technology, Beijing 100081, \\ China;

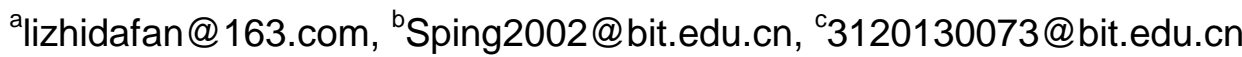

Keywords: data transmission, wireless network, telemetry.

\begin{abstract}
This paper proposes a telemetry data transmission method based on wireless multi-hop network. This method is based on a transmission network constituted by several wireless nodes and adopts modified ZigBee wireless communication protocol. In this method, the telemetry terminal and the gateway will coordinate the whole transmission network automatically during the data transmission and the order transmission. According to the experiment results, this transmission method can guarantee stable and high-efficient transmission and can be used in many telemetry data transmission scenario.
\end{abstract}

\section{Introduction}

People always telemeter the target to get its working condition when the target is in the distance or extreme conditions. Data transmission is one of the key technical of telemetry because the accuracy and real-time of data transmission determines whether we can accomplish the telemetry task [1, 2]. In the field of telemetry, wireline transmission and point-to-point wireless transmission are used to transfer data traditionally. Wireline transmission is stable but its installation need high costs and cumbersome steps, and point-to-point wireless transmission has distance limitation reasoned by signal attenuation.

Wireless multi-hop self-organizing network can realize inter-node communication without base stations. This network has a good expansibility and low power consumption, can be applied in high temperature, low temperature, high-altitude and some other limit environment [5]. Given the above, this paper proposes telemetry data transmission method based on wireless multi-hop network. In this method, wireless network constituted by several data reception nodes will be the data reception device. Compared with point-to-point wireless transmission, this method can extend the range of receiver significantly because every node has the same communication distance as traditional point-to-point wireless communication.

\section{System Design}

\subsection{Hardware.}

This transmission network is constituted by several wireless nodes with wireless communication module, and gets data from the data receive module via serial port. Wireless node is composed of FPGA, wireless communication module, micro-SD card and other supporting components. We adopt Cyclone II FPGA from Altera Company, which is one of the fastest chip in low-cost fields. We choose Atmel Company's ATZB-S1-256-X module as our wireless communication module, which has Atmega256X integrated microchip inside and SMA connector on it. Atmega256X integrated microchip is an 8-bit microcontroller with low power $2.4 \mathrm{GHz}$ transceiver for ZigBee and IEEE 802.15.4, and its protocol stack is open source, which made our design of communication tactics more freely. Besides, Atmega256X integrated microchip can elevate its transmission rate to 2Mb/s, which can help us further improve the efficiency of transmission. 
The block diagram of wireless node is shown in Fig. 1. FPGA is the control center of the wireless node and undertake functions of data processing and statistical analysis. When data receive module sends telemetry data, FPGA will write the data to micro-SD card in fixed format. When the wireless node receive data transmission instruction from the telemetry terminal, FPGA will pull out the telemetry data from micro-SD card and send it to the wireless communication module. We choose 2.4 $\mathrm{GHz}$ Swivel SMA Antenna to finish our studies, people can use high-power microwave antenna to fulfil telemetry task.

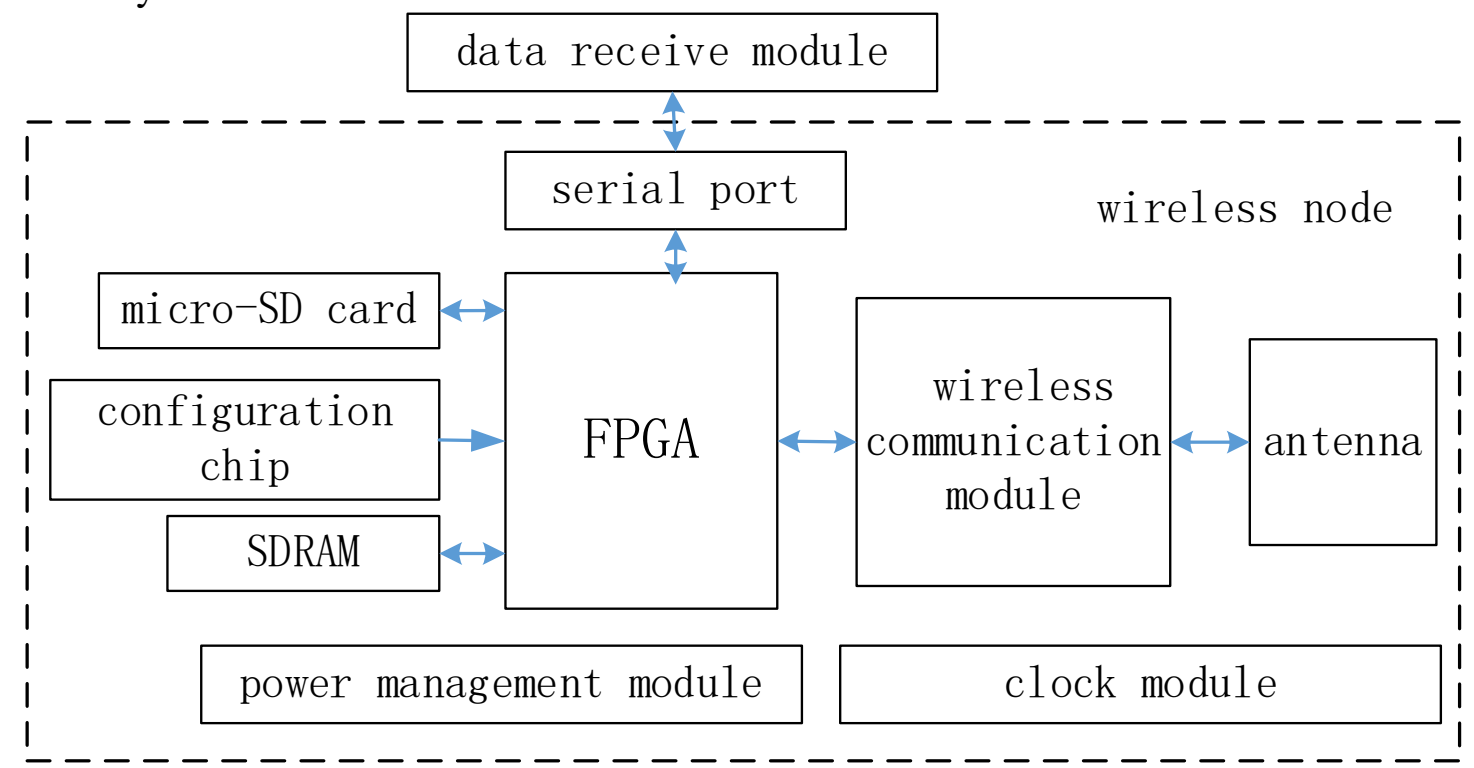

Fig. 1 The block diagram of wireless node

\subsection{Transport Mechanism.}

All wireless node will form a wireless multi-hop network after turned on, and this network is connected by wireless communication module. This wireless multi-hop network communicate with telemetry terminal by a gateway, which has a wireless communication module and a serial port. Topology relationship between telemetry terminal, gateway and wireless nodes is shown in Fig. 2, and this three parts constitute the telemetry network. Wireless nodes send their state to telemetry terminal periodically, while receive all kinds of instructions such as reset, re-configuration and data transmission instruction from telemetry terminal via the wireless multi-hop network.

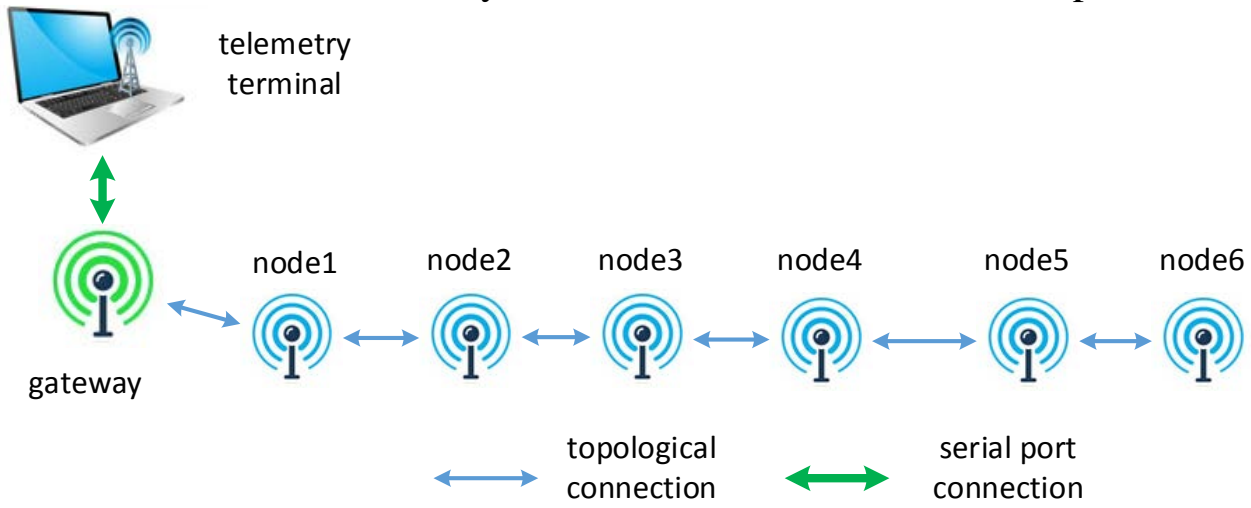

Fig. 2 Topology relationship in telemetry network

As mentioned above, when telemetry starts, data receive module will send telemetry data to FPGA via serial port. At the same time, FPGA will start SD card driver and write telemetry data into micro-SD card in SD mode, and the data rate of SD mode is up to $10 \mathrm{Mb} / \mathrm{s}$, which can meet most telemetry's rate requirement. When wireless node is receiving data, it will also inform the telemetry terminal, so people can see every node's working state clearly.

When the data reception is complete, data transmission instruction will be sent from the telemetry terminal. All wireless nodes will collaborate with each other to send telemetry data back to telemetry terminal. Transmission strategy is step-by-step and point-to-point to avoid the accumulation of packet 
loss and invalid occupy of network resource. The first two transmission cycle's transmission relation is shown in Fig. 3, wireless nodes form to 3 pairs and start super-frame transmission. As the two nodes in one pair, one is sending data from its micro-SD card and the other one receive it and write it into micro-SD card. After several transmission cycles, all the telemetry data will be sent to telemetry terminal.

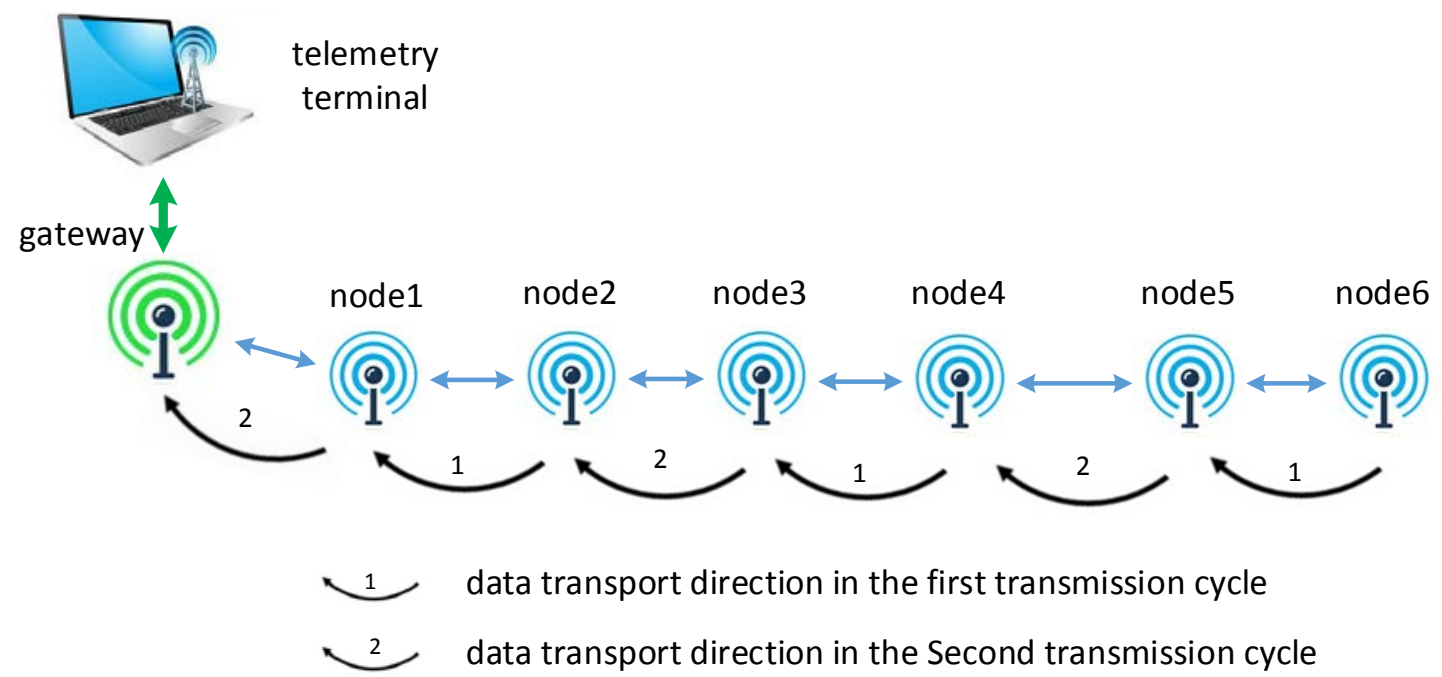

Fig. 3 Schematic of data transmission strategy

Gateway initiate super-frame transmission and specify which node send or receive data. The steps of transfer data in super-frame state is shown in Fig. 4. When wireless nodes is in the super-frame state, their transfer rate will be switched to $2 \mathrm{Mb} / \mathrm{s}$ and their frame header will be omitted to enhance its transfer data rate. Every transmission cycle has several super-frame transmission pairs. After several transmission cycles, all the telemetry data will be sent to telemetry terminal.

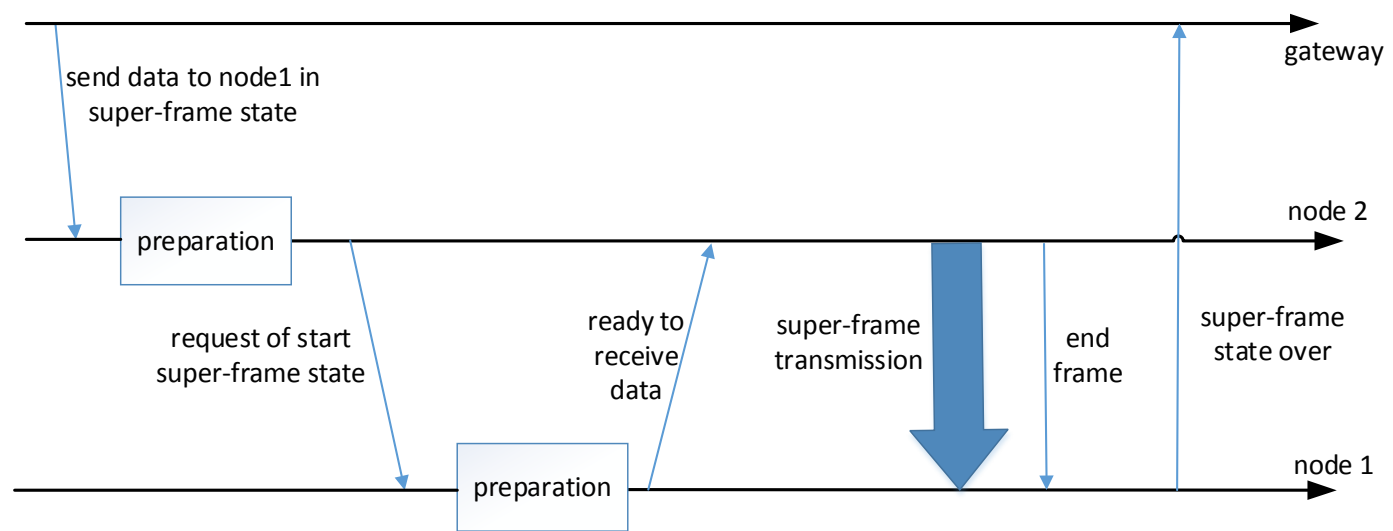

Fig. 4 process of super-frame transmission

After receiving telemetry data from wireless nodes, the telemetry terminal will store the telemetry data in formation based on telemetry data's time information or serial number. Besides, if telemetry data sequence number the telemetry terminal can calculate packet loss rate and generates a brief report.

\section{Experimental Results}

In order to verify our scheme, we carried out practicality experiment. We reduced the output power and sensitivity of wireless commutation module to simulate telemetry experiment. According to the law of electromagnetic wave attenuation, this method can be regarded as a reduced scale model of telemetry.

We set the out power of wireless commutation module to $-2.5 \mathrm{dBm}$, and sensitivity to $-86 \mathrm{dBm}$, and test its reliable communication distance which showed 10 meters. Eight wireless nodes were placed 
on the open ground with interval of 10 meters and then be turned on one by one. The working state and the topology of wireless node were shown on the telemetry terminal. 5 seconds later, all the wireless node are in proper function and formed a linear topology like the topology in Fig. 3.

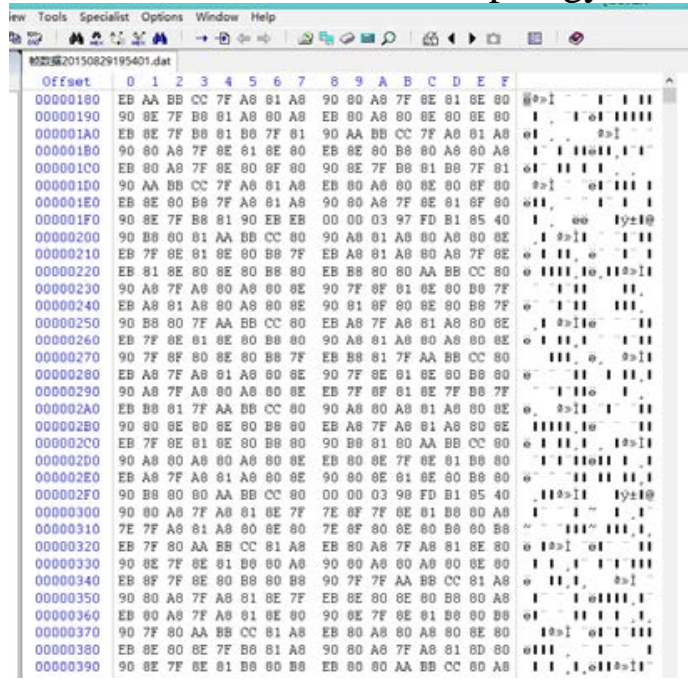

Fig. 5 Test data received from wireless nodes

We sent $5 \mathrm{Mb}$ of data to every node by data receive module, and noticed the working state change of wireless nodes when we sent. After that, we sent data transmission instruction from telemetry terminal to every wireless node. 5 minutes later, all the data was sent to the telemetry terminal with $3 \%$ data loss.

\section{Summary}

This paper proposes a telemetry data transmission method based on wireless multi-hop network, which realizes the scalability of wireless telemetry. Test results proved that the application of multi-hop network in telemetry can improve the transmission distance significantly. Thanks to the adoption of super-frame transmission strategy, the network overcomes the low rate defect of the ordinary ZigBee network, and makes it more suitable for most tests.

However, our strategy adopt single channel, high depth topology, which may cause more data loss when one node is failure.

\section{Acknowledgement}

Our research is partially supported by National Natural Science Foundation of China (Grant No.61202433). We also acknowledge the helpful suggestions from the reviewers.

\section{References}

[1]. DONG Hang, SUN Fayu, ZHENG Xiaoyan, et al. Telemetry Transmission Method Based on IEEE 802.11b [J]. Journal of Detection \& Control. Vol. 37(2015) No.3,p.74-77.

[2]. Sun Fayu. Ordnance Telemetry Development Prospect and Tendency[J]. COMPUTER MEASUREMENT \& CONTROL. Vol.16(2008)No.10,p.1371-1372.

[3]. TANG Qiang, LUO Haitao, ZHENG Xiaoyan, et al. High Speed Wireless Network Based on Wireless Sensor Node[J]. Journal of Detection \& Control. Vol.36(2014) No.2,p.69-73.

[4]. Gao Yul, Zhu Qiuming, Luo Yanqiang. Design and Implementation of Telemetry Instrument Automatic Test System[J]. Journal of Data Acquisition \& Processing. Vol.25(2010) No.5,p.672-676. 
[5]. Yuan Yi, Su Honggen. THE STUDY OF WIRELESS NETWORK APPLICATIONS BASED ON ZIGBEE TECHNOLOGY[J]. COMPUTER APPLICATIONS AND SOFTWARE. Vol.21(2004) No.6,p.89-91. 